\title{
Implementation of Digital Micro Fluidic Biochip with Machine Learning for Drug Development
}

\author{
A. Obulesu ${ }^{1}$, P. Arulprakash ${ }^{2}$, D. Vijaya Saradhi ${ }^{3}$, K. Mahesh Kumar ${ }^{4}$ and Kamepalli Sujatha \\ ${ }^{1}$ Department of Artificial Intelligence, Vidya Jyothi Institute of Technology (Autonomous), Hyderabad, TS, India \\ ${ }^{2}$ Department of CSE, Rathinam Technical Campus, Coimbatore, TN, India \\ ${ }^{3}$ Department of ECE, Malineni Perumallu Educational Society Group of \\ Institutions, Guntur, Jntu-K, Andhra Pradesh, India \\ ${ }^{4}$ Department of CSE, Koneru Lakshmaiah Education Foundation Vaddeswaram, Guntur 522502 \\ ${ }^{5}$ Department of Information Technology, VFSTR Deemed to be University, Guntur, Andhra Pradesh, Inida \\ Corresponding author email: avuku06@gmail.com
}

\section{ABSTRACT}

Machine learning can be very useful in a variety of applications, such as data mining and pattern recognition. In the fields of bioinformatics, and other types of pharmaceutical research, machine learning techniques have been widely used. In the biopharmaceutical field, drug discovery and development are becoming increasingly important. Current drug discovery, on the other hand, is slow, costly, incompetent, and tend toerrors. The digital micro fluidic chip has the potential to be a very valuable technique for drug detection. Furthermore, machine learning algorithms were used to aid in the detection of new drugs. Pharmaceutical companies are currently suffering from low production costs that could be addressed by automating the drug development. A variety of techniques, including drug target design and pharmaceutical research, are now regularly enhanced by machine learning algorithms. As a result of techniques such as high-throughput testing and simulation methods of databases used for lead and target discovery, machine learning and deep learning techniques have become more reliable. Researchers have also used machine learning algorithms, such as deep learning methods, for drug discovery applications; digital microfluidic platforms will be utilized as a supplement to existing drug development methodologies that use machine learning algorithms. Digital microfluidics will supplement existing high throughput screening techniques, offering benefits in terms of performance, sample, and state of health replicates. Computer-aided design techniques in digital micro fluidics can be used to obtain information\& machine learning algorithms can be used to analyze it, in order to enhance efficiency and durability.

\section{KEY WORDS: MACHINE LEARNING, DIGITAL MICROFLUIDICS, DRUG DISCOVERY.}

\section{INTRODUCTION}

In recent times, with the genome sequencing project's products assisting in the discovery of many serious disease targets at which differential diagnoses could be directed,

Biosc Biotech Res Comm P-ISSN: 0974-6455 E-ISSN: 2321-4007

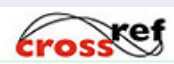

Identifiers and Pagination

Year: 2021 Vol: 14 No (7) Special Issue

Pages: $457-462$

This is an open access article under Creative

DOI: $h t t p: / / d x$.doi.org/10.21786/bbrc/14.7.95
Commons License Attribn 4.0 Intl (CC-BY). all of the major drug manufacturers have heavily invested in regular ultra-High Mass Spectrometry (uHTS) for immense proprietors of 'drug-like' particles supported by looks like a real investigation. Building a novel medication is complicated, time intense, \& expensive process, and because of high cost of late-stage drug aspirant failures, uHTS results include obligations such as probable effects as well and medical benefits, and computational techniques will be utilized to aid \&accelerate the drug design course. Faster processes for identifying chemical amalgams among desired biological possessions \& optimizing their framework to produce effective drugs would undoubtedly benefit the drug discovery and development process. For example, structure activity relationships (QSAR) models have the
Article Information

Received: $10^{\text {th }}$ Aug 2021

Accepted after revision: $15^{\text {th }}$ Oct 2021 
opportunities to resolve several major bottlenecks in drug discovery (Lipinski, 2014; Leeson, 2014; Li-Preclinical et al., 2015; Hansch et al., 2019).

The systematic, sequential process of identifying and modifying chemical composition in order to attain expected results adjacent to therapeutic properties along with a specific infection is referred to as methodology and discovery. The proposed methods for drug discovery begin with scientists learning about the inherent and molecular basis of one infection \& then applying that knowledge to choose potential treatment. aim by testing thousands of substances against the objectives using high bandwidth evaluation to recognize those substances that hit the targets (HTS). After understanding the effects of the tested compounds and identifying commonly applied structural elements, novel materials contain those structural elements are produced, likely to result in a drop in the rate of substances. Finally, the recognized gives rise is developed further to meet pharmacodynamic restrictions such as absorption, as well as to increase efficacy and truthfulness while decreasing negative impacts and toxic effects, a practice called as combination, allocation, metabolism, removal, and toxic effects properties (Segall et al., 2018).

Machine learning techniques were initially found to be extremely useful in drug development, screening, and recognizing applicant particles, as well as research on measurable structure movement associations (QSAR), that will distinguish among collection of substances which were effective towards this particular genetic receptor includes a wide range of techniques or methodologies for a wide range of drug design processes, including such decision trees, lazy trying to learn, k-nearest neighborhood, Naive baye's methods, Probability distribution procedures, artificial neural networks, artificial callousscheme, particle-swarm optimization, Ant colony optimization, cuckoo search, support vector machines, and kernel. (Bishop, 2006; Duch, 2007; Mohri, 2009; Marsland, 2009; Chin, 2012; Gertrudesa, 2012; The supervised learning Burke, 2014). These are their given names:

i)Virtual screening: Machine learning methods are used in screening to grade or sort proteins depending on multiple properties. Machine learning techniques improve pattern matching when only a few source compounds are provided - particularly in ligand dependent essential viewing, somewhere no structural data concerning the objective is accessible. Furthermore, machine learning techniques can be used to generate a variety of compounds that can be used as contribution for viewing (Hert et al., 2006; Schneider et al., 2008).

ii). Structure activity relationship (QSAR) and structure activity relationship (QSPR) models: The QSAR and QSPR models are mathematical methods which are used to infer relationships among organic compounds and their microbial properties or physical characteristics Kubiny et al., (2003). iii). Protein, purpose, and communication forecasting: Machine learning techniques have discovered vast application in biological tasks such as protein identification, automated reasoning forecasting, and interacting proteins categorization.

Machine learning models, as well as the introduction of the QSAR field of research, are becoming more common in the analysis of structure-activity data. We will only concentrate on machine learning techniques in QSAR application domains Malik et al., (2013). Machine learning methods use statistical and mathematical order to derive information from novel data in order to produce a collection of policies, purposes, or programmers which permit someone to forecast the characteristics of novel things which were not in knowledge base. A training set is officially assigned with the task of choosing the best premise to support the information.

Machine learning techniques were now being quickly applied to a variety of fields, and the total majority of publications written is going to increase, especially in recent years. The implementation of these methods to existing pharmacological pioneer work may include a positive effect on such pains. However, based on research, drugrelated machine learning papers are still in their infancy. Drug development is an uncertain effect that necessitates a multidimensional research space. When compared to macroscale procedures, the digital microfluidic platform employs smaller sample sizes, which reduces sample costs.

Literature Survey: In this segment, we will go over the history of how machine learning methods were used in pharmacological exploration. The Bayes theorem, which is commonly used with data science, is also popularly utilized during drug development. Semerwalkino et al., Ekins et al., (2015) discussed the integration of genomics \&boost growth and presented a technique for selecting substances for testing using three dual event Bayesian models. Following that, the samples were screened, evaluated, and optimized. This method can be used to discover new treatments like malaria. Lavecchia et al., (2015) spoke about the use of machine learning techniques in Ligand dependent Virtual program (LBVS). Their investigation had been effective in categorizing molecules and forecasting new active particles due to advancements and increases in community, vast biological and chemical data. The Bayesian Network technique has been used to predict chemical biological activities and potency, as well as protein attack and biosynthetic pathway categories. This system was accurate in explaining whether or not a given compound is active.

A standout amongst other regulated learning strategies, Support Vector Machine (SVM), has been prevalently utilized in drug improvement. Warmuth et al., (2003) endeavored to tie substances from a colossal assortment to a specific supplement in a couple of rehashes or cycles as plausible. The most pivotal system was characterized as the greatest rate subspace produced by SVMs. By and large, their information exhibited how SVMs can be helpful in distinctive important data from arbitrary data. This is additionally really the situation for powerful learning circumstances in which information was recovered during investigations to help with the improvement of results. 
Byvatov et al., (2001) broke down the exactness of a SVM and a Neural Network (NN) strategy on drug arrangement versus non order. The overview gave a complete report wherein the SVM has been displayed to yield unexpected outcomes in comparison to the NN models, yet with a minuscule edge of differentiation. Liu et al., (2014) broke down the precision of five element determination methods: at long last discovered, information combination, a chisquare test, relationship coefficient, and GSS co - effective. Then, at that point, on the sets of element vectors and on the sets with all elements, a Naive Bayesian classifier and a SVM classifier were tried. Shockingly, the SVM played out the best when all components were thought of, while the Bayes Classifier performed best when a couple of elements were thought of.

Another widely used technique in this field is the neural network (NN) technique. Menden et al., (2013) demonstrated how NN could be used to forecast how a particular tumor will react to drug treatment. To accomplish this, the NN forecasted how cancers would interact to drug therapy depending on gene expression characteristics of the cell lines\& chemical goods of the known drug. Data was analyzed using $8 \%$ increase cross-validation and a nutrient multi - layer perceptron. Training algorithm was used for training the database schema. Geturtrure et al., Lavecchia et al., (2015) stated why drug discovery is so difficult, and then evaluated different techniques and their efficacy, like conscience maps, multiple layer perception, Probabilistic neural networks, neutralize neural networks, and support vector machine.

Other machine learning methods, such as unsupervised learning methods and data analysis method implementations, are also used in this research. Armugam et al., (2016) used active education to determine the effect of 59 chemicals on the subcellular of 59 proteins despite of conducting $29 \%$ of all probable trials. This work uncovered a big data approach to notice lethal consequences that would prevent a drug from testing on humans before the costly scientific experiment period. PrOCTOR is a method inspired by the Baseball method, which was popularized in baseball.

The researchers trained their machine to analyze every drug using 59diversecharacteristics in order to determine its scientific suitability. Huo Damn et al., Ding et al., (2013) planned a Nearest Neighbor-like correlation method to determine what will be the most effective in forecasting new drugs and latest target drug connections. The drug $\&$ target spaces were primary projected into two lowdimensional spaces using kernels. Low-dimensional spaces were then used to estimate drug-target interactions. This was inefficient because it used three matrices, each of which had random values seeded into it. Giguere et al., (2015) proposed a graph theory-based drug discovery approach. Because of the kernel requirements, this technique is claimed to be compatible with learning algorithms such as SVM, SVR, Ridge dropping, \& Gaussian procedure, but will not NNs.

Digital Micro fluidic Biochip Methodology: A digital microfluidic biochip (DMFB) manipulates discrete particles samples collected and materials on a 2 dimensional attractive grid of wires using the lectrowetting-on-dielectric principle. The distinct fluid is resting on a metallic substrate over an anode on an anode array. Activities can be carried out by using voltage sensor scenes. Figure 1 defines the proposed unit cell of an electronic microelectronic biochip (Tang et al., 2018).

Figure 1: Structure of a DMFB: (a) unit cell side view (b) a general-purpose DMFB (Tang et. al. 2018).

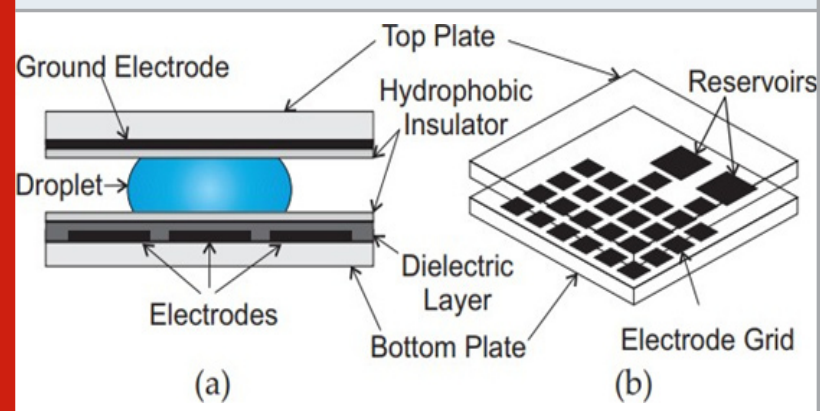

The DMFB is made up of two pieces of glass. The lower part crystal plate contains a variety of application-specific control membranes, while the marble backsplash plate contains a floor cathode. To reduce surface wet ability and amplify capacitance among the control electrodes \& the droplet, a lipophilic insulating insulator is introduced into the two plates. Applying a current to a cathode near the droplet while removing the cathode beneath the liquid can cause the droplet to go towards the charge electrode (Majunder et al., 2011).

Before being released into watersheds, droplets can be processed on an anode, identified by sensors, transferred between wires, mixed with other particles, or joined from supplying ports. I/O reservoirs on the processor's perimeter perform dispensing and output operations, and sensors are placed in specific locations on the biochip. The DMFB manages multipurpose processor applications and employs Tools for computerized chip synthesis and test protocol enhancement. Based on the synthesis results, a microcontroller was also worn to manage the voltage of electrodes.

By varying the patterns of control voltage activation, basic analyze activities such as discharging, transit, blending, combining, warming, fermentation, and dividing can be performed on the chip. Moving two particles to the same cell and rotating them around a pivot, for example, can be used to perform a mixing operation (Paik et al., 2003). Because fundamental procedure will be performed anyplace on the array, the DMFB allows for dynamic reconfigurability. Also. DMFBs use optical detectors, such as LEDs, to monitor colorimetric bioassays Srinivasan et al., (2004). A variety of bio-assays are performed using digital micro fluidic platforms. A robotized configuration stream is utilized to arrange bioassays on chip into programming to enact the anodes (Grissom et al., 2012). 
Acceleration of Drug Discovery by Machine Learning: As a substitute to conventional technique, computational techniques were used in pharmaceutical research. By introducing new runs from relevant theories library services, the innovative methods can reduce expenses accelerate the process necessary to produce latest drug candidates. Machine learning methods, such as screening, were used to develop alternative QSAR models for the drug development process (VS). Drug development must take advantage of recent innovations in elevated screening (HTS). The conventional QSAR method, on the other hand, is incompatible with the recent big data approach (Zhang et al., 2017).

Machine learning, particularly in neural networks (ANNs) \& big data (DL), offer an exciting ability in drug development. Figure 4 defines the proposed framework of an ANN, which is motivated by the structure of the brain. The contribution, outcome, and hidden units are the three basic levels in an ANN. Because the conventional ANN will have flaws, it was restored by certain methodologies for example RF and SVM. However, machine learning has impacted an increasing number of ANN algorithms. Because feed-forward neural networks are coupled with a number of concealed units, machine learning is indeed derived from conventional ANN (Zhang et al., 2017).

Machine learning techniques can be implemented to drug discovery data. Machine learning can create organized information \& answer issues in drug discovery that really are common to microfluidic devices. Microelectronic advancements have compensated drug development with massive amounts of data, but these systems often do not have the capability to process efficiently. Deep learning has been using detailed detection of input data, including such pictures and scenes, in recent times to anticipate complicated yields significant Riordon et al., (2018).

Learning machines can now be trained in arbitrarily defined aim, ligand goal, \& ligand result connections. Based on variables \&drug devise objectives, such algorithms can detect hidden patterns in particles. It is possible to generate the desired trends for further organic synthesis, evaluation, and verification. Mark free cell characterization, for instance, is favorable in drug revelation and improvement since it maintains a strategic distance from the adverse consequences of staining test and reagent on cell flagging. Proposed troupe learning, a mark free cell arrangement strategy, just as enormous information imaging (Chen et al., 2016; Schneider et al., 2017).

Machine learning implementations in pharmaceutical research are summarized as follows: (1) introduction of novel drug particles, (2) protein engineering, (3) genomic statistical analyses, and (4) pharmacology modelling. For example, Pereera et al., suggested a VS method based on machine learning; they docked with 2950 receptors and 59 synapses and compared their performance to 95316 detonators. Their results demonstrated the advantages of their technique by achieving the finest achievement for Dimensionality 59 receptor sites. Furthermore, PD modeling is important for determining the stronger connection among medications and targets (such as proteins). Wen et al., utilized machine learning to anticipate medications in a recent study. They utilized 2879568 medication protein association sets with supported medications and targets. Their discoveries featured the developing capability of AI for finding new original medication experiences (Zhang et al., 2017).

Besides, computerized microfluidics can result to treatment robotization using CAD devices. Computer aided design calculations, then again, don't investigate library information, nor do they grow our screening library from a great many substances, nor do they add a layer of beneficial data that can help researcher's configuration new trials. In fact, by joining AI and microfluidic advancements, we could undoubtedly accomplish the best medication revelation plans and systems.

Figure 2: An example of a neural network (NN): (a) a fundamental NN is made up of the contribution, production, \&concealed units. (b) Using an input image, the hidden unit's production parameters are displayed from the input values.

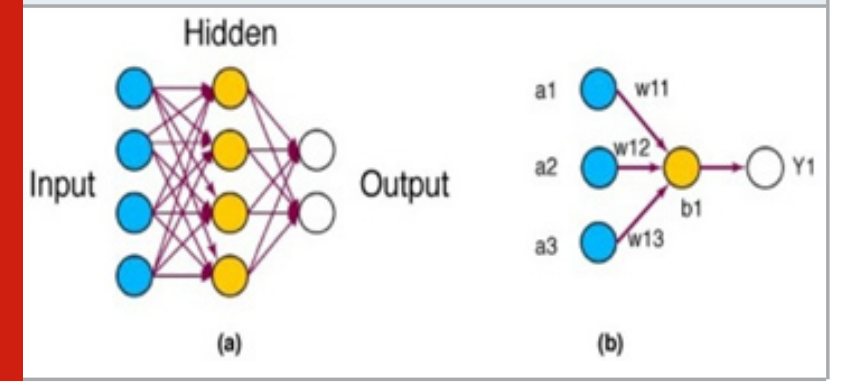

Microfluidics is used in several studies which introduces a technique for decoding experimental circumstances in microfluidic drop analyzes and employs machine learning endorsed imaging techniques. The method relies on the coencapsulation of polyethylene crystals with microbial. In addition, as a high throughput cell evaluation environment, a microfluidic device is tried to introduce. The improvement of a liver particular cells phenotypic-biomarker test with singular cell goal and approval with two unique malignant growth tissue tests for forecast of post-careful pathology was accounted for by analysts Specialists utilized support learning calculations, just as Deep Q-Networks, to plan verbose controllers for microfluidic gadgets in their work (both constant stream and portioned stream microfluidic stages). They stressed the meaning of fusing a control framework into raised microfluidic gadgets (Dressler et al., 2018; Manak et al., 2018; Svensson et al., 2019).

Furthermore, deep neural structures will be urbanized to singular science, cognitive science, and computational biology; methods that have been effective in micro microfluidic devices and pertinent architectures will be instituted. It is intended to examine simple unorganized cases up to more complicated structures. To categories samples with multiple densities of DMFB, a human brain able to carry unstructured inputs will be used; various imaging techniques will be used to sample images. The new 
DMFB-ML platform, like our preceding sample collection DMFB design, will be intended for drug development velocity, but it would also be used in other biomedicine areas; this would be merged with micro systems for molecule identification. In addition, a protein crystallization DMFB-ML platform for determining protein content would be devised (Riordon et al., 2018).

\section{CONCLUSION}

Machine learning techniques were now being quickly applied to a variety of fields, and the total majority of publications written is going to increase, especially in recent years. The implementation of these methods to existing pharmacological pioneer work may include a positive effect on such pains. However, based on research, drugrelated machine learning papers are still in their infancy. Drug development is an uncertain effect that necessitates a multidimensional research space. When compared to macroscale procedures, the digital microfluidic platform employs smaller sample sizes, which reduces sample costs. Furthermore, because they are reconfigurable, automated, and scalable, these online technologies outperform continuous flow microfluidic devices. Researchers have also used machine learning algorithms, such as deep learning methods, for drug discovery applications; digital microfluidic platforms will be utilized as a supplement to existing drug development methodologies that use machine learning algorithms. Digital microfluidics will supplement existing high throughput screening techniques, offering benefits in terms of performance, sample, and state of health replicates.

\section{REFERENCES}

Bishop, C.M., (2006). Pattern recognition. Machine learning, 128(9).

Burke, E.K., Burke, E.K., Kendall, G. and Kendall, G., (2014). Search methodologies: introductory tutorials in optimization and decision support techniques. Springer.

Byvatov, E., Fechner, U., Sadowski, J. and Schneider, G., (2003). Comparison of support vector machine and artificial neural network systems for drug/nondrug classification. Journal of chemical information and computer sciences, 43(6), pp.1882-1889.

Ding, H., Takigawa, I., Mamitsuka, H. and Zhu, S., (2014). Similarity-based machine learning methods for predicting drug-target interactions: a brief review. Briefings in bioinformatics, 15(5), pp.734-747.

Dkhar, L.K., Bartley, J., White, D. and Seyfoddin, A., (2018). Intranasal drug delivery devices and interventions associated with post-operative endoscopic sinus surgery. Pharmaceutical development and technology, 23(3), pp.282-294.

Duch, W., Swaminathan, K. and Meller, J., (2007). Artificial intelligence approaches for rational drug design and discovery. Current pharmaceutical design, 13(14), pp.1497-1508.

Ekins, S., Madrid, P.B., Sarker, M., Li, S.G., Mittal, N., Kumar, P., Wang, X., Stratton, T.P., Zimmerman, M., Talcott, C. and Bourbon, P., (2015). Combining metabolite- based pharmacophores with bayesian machine learning models for Mycobacterium tuberculosis drug discovery. PloS one, 10(10), p.e0141076.

El-Telbany, M.E., (2014). What quantile regression neural networks tell us about prediction of drug activities. In 2014 10th International Computer Engineering Conference (ICENCO) (pp. 76-80). IEEE.

Giguere, S., Laviolette, F., Marchand, M., Tremblay, D., Moineau, S., Liang, X., Biron, É. and Corbeil, J., (2015). Machine learning assisted design of highly active peptides for drug discovery. PLoS computational biology, 11(4), p.e1004074.

Grissom, D. and Brisk, P., (2012). Path scheduling on digital microfluidic biochips. In DAC Design Automation Conference 2012 (pp. 26-35). IEEE.

Hansch, C., (1969). Quantitative approach to biochemical structure-activity relationships. Accounts of chemical research, 2(8), pp.232-239.

Hert, J., Willett, P., Wilton, D.J., Acklin, P., Azzaoui, K., Jacoby, E. and Schuffenhauer, A., (2006). New methods for ligand-based virtual screening: use of data fusion and machine learning to enhance the effectiveness of similarity searching. Journal of chemical information and modeling, 46(2), pp.462-470.

Kubinyi, H., (2003). QSAR in drug design. Handbook of Chemo informatics: From Data to Knowledge in 4 Volumes, pp.1532-1554.

Lavecchia, A., (2015). Machine-learning approaches in drug discovery: methods and applications. Drug discovery today, 20(3), pp.318-331.

Leeson, P.D., Davis, A.M. and Steele, J., (2004). Druglike properties: guiding principles for design-or chemical prejudice? Drug Discovery Today: Technologies, 1(3), pp.189-195.

Lipinski, C.A., (2004). Lead-and drug-like compounds: the rule-of-five revolution. Drug discovery today: Technologies, 1(4), pp.337-341.

Li, A.P., (2005). Preclinical in vitro screening assays for drug-like properties. Drug Discovery Today: Technologies, 2(2), pp.179-185.

Majumder, M., Hansda, K. and Roy, S., (2011). A novel single-fault detection technique of digital microfluidic biochip. Int. J. Comput. Sci. Appl, 1, pp.92-95.

Malik, J.K., Soni, H. and Singhai, A.K., (2013). QSARApplication in Drug Design. International Journal of Pharmaceutical Research \& Allied Sciences, 2(1).

Marsland, S., (2009). Machine learning, an algorithmic perspective, chapman \& hall/crc machine learning \& pattern recognition. CRC, Boca Raton, Fla.

Manak, M.S., Varsanik, J.S., Hogan, B.J., Whitfield, M.J., Su, W.R., Joshi, N., Steinke, N., Min, A., Berger, D., Saphirstein, R.J. and Dixit, G., (2018). Live-cell phenotypic-biomarker microfluidic assay for the risk stratification of cancer patients via machine learning. Nature biomedical engineering, 2(10), pp.761-772.

Menden, M.P., Iorio, F., Garnett, M., McDermott, U., Benes, C.H., Ballester, P.J. and Saez-Rodriguez, J., (2013). 
Machine learning prediction of cancer cell sensitivity to drugs based on genomic and chemical properties. PLoS one, 8(4), p.e61318.

Mohri, M., Rostamizadeh, A. and Talwalkar, A., (2012). Foundations of machine learning.

Momtahen, S., Al-Obaidy, F. and Mohammadi, F., (2019). Machine Learning with Digital Microfluidics for Drug Discovery and Development. In 2019 IEEE Canadian Conference of Electrical and Computer Engineering (CCECE) (pp. 1-6). IEEE.

Momtahen, S., Al-Obaidy, F. and Mohammadi, F., (2019). Machine Learning with Digital Microfluidics for Drug Discovery and Development. In 2019 IEEE Canadian Conference of Electrical and Computer Engineering (CCECE) (pp. 1-6). IEEE.

Naik, A.W., Kangas, J.D., Sullivan, D.P. and Murphy, R.F., (2016). Active machine learning-driven experimentation to determine compound effects on protein patterns. Elife, 5, p.e10047.

Paik, P., Pamula, V.K. and Fair, R.B., (2003). Rapid droplet mixers for digital microfluidic systems. Lab on a Chip, 3(4), pp.253-259.

Riordon, J., Sovilj, D., Sanner, S., Sinton, D. and Young, E.W., (2019). Deep learning with microfluidics for biotechnology. Trends in biotechnology, 37(3), pp.310324.

Schneider, G. and Baringhaus, K.H., (2008). Molecular design: concepts and applications. John Wiley \& Sons.

Schneider, G., (2018). Automating drug discovery. Nature reviews drug discovery, 17(2), pp.97-113.

Srinivasan, V., Pamula, V.K. and Fair, R.B. (2004). An integrated digital microfluidic lab-on-a-chip for clinical diagnostics on human physiological fluids. Lab on a Chip, 4(4), pp.310-315.

Stephenson, N., Shane, E., Chase, J., Rowland, J., Ries, D., Justice, N., Zhang, J., Chan, L. and Cao, R., (2019). Survey of machine learning techniques in drug discovery. Current drug metabolism, 20(3), pp.185-193.

Svensson, C.M., Shvydkiv, O., Dietrich, S., Mahler, L., Weber, T., Choudhary, M., Tovar, M., Figge, M.T. and Roth, M., (2019). Coding of experimental conditions in microfluidic droplet assays using colored beads and machine learning supported image analysis. small, 15(4), p. 1802384 .

Tang, J., Ibrahim, M., Chakrabarty, K. and Karri, R., (2018). Tamper-resistant pin-constrained digital microfluidic biochips. In 2018 55th ACM/ESDA/IEEE Design Automation Conference (DAC) (pp. 1-6). IEEE. Warmuth, M.K., Liao, J., Rätsch, G., Mathieson, M., Putta, S. and Lemmen, C., (2003). Active learning with support vector machines in the drug discovery process. Journal of chemical information and computer sciences, 43(2), pp.667-673.

Yee, L.C. and Wei, Y.C., (2012). Current modeling methods used in QSAR/QSPR. assessment, 10, p.11.

Zhang, L., Tan, J., Han, D. and Zhu, H., (2017). From machine learning to deep learning: progress in machine intelligence for rational drug discovery. Drug discovery today, 22(11), pp.1680-1685. 